ARTICLE

\title{
Phosphorylation of BLUS1 kinase by phototropins is a primary step in stomatal opening
}

Atsushi Takemiya ${ }^{1}$, Naoyuki Sugiyama ${ }^{2 \dagger}$, Hiroshi Fujimoto ${ }^{1}$, Toshifumi Tsutsumi ${ }^{1}$, Shota Yamauchi ${ }^{1}$, Asami Hiyama', Yasuomi Tada ${ }^{3}$, John M. Christie $^{4} \&$ Ken-ichiro Shimazaki ${ }^{1}$

Opening of stomata in the plant facilitates photosynthetic $\mathrm{CO}_{2}$ fixation and transpiration. Blue-light perception by phototropins (phot1, phot2) activates the plasma membrane $\mathrm{H}^{+}$-ATPase, causing stomata to open. Here we describe a regulator that connects these components, a Ser/Thr protein kinase, BLUS1 (BLUE LIGHT SIGNALING1), which mediates a primary step for phototropin signalling in guard cells. blus1 mutants identified by infrared thermography result in a loss of blue light-dependent stomatal opening. BLUS1 encodes a protein kinase that is directly phosphorylated by phot1 in vitro and in vivo at Ser-348 within its C-terminus. Both phosphorylation of Ser-348 and BLUS1 kinase activity are essential for activation of the $\mathrm{H}^{+}$-ATPase. blus1 mutants show lower stomatal conductance and $\mathrm{CO}_{2}$ assimilation than wild-type plants under decreased ambient $\mathrm{CO}_{2}$. Together, our analyses demonstrate that BLUS1 functions as a phototropin substrate and primary regulator of stomatal control to enhance photosynthetic $\mathrm{CO}_{2}$ assimilation under natural light conditions.

\footnotetext{
${ }^{1}$ Department of Biology, Faculty of Science, Kyushu University, 6-10-1 Hakozaki, Fukuoka 812-8581, Japan. ${ }^{2}$ Institute for Advanced Biosciences, Keio University, Tsuruoka, Yamagata 997-0017, Japan. ${ }^{3}$ Life Science Research Center, Institute of Research Promotion, Kagawa University, 2393 Ikenobe, Miki-cho, Kita-gun, Kagawa 761-0795, Japan. ${ }^{4}$ Institute of Molecular, Cell and Systems Biology, College of Medical, Veterinary and Life Sciences, University of Glasgow, Glasgow G12 8QQ, UK. † Present address: Graduate School of Pharmaceutical Sciences, Kyoto University, Sakyo-ku, Kyoto 606-8501, Japan. Correspondence and requests for materials should be addressed to K.S. (email: kenrcb@kyushu-u.org).
} 
S ophisticated control mechanisms optimize stomatal aperture and allow land plants to occupy habitats in ever changing environments ${ }^{1,2}$. Stomatal aperture is regulated by light, water status, the phytohormone abscisic acid, $\mathrm{CO}_{2}$ and other environmental factors, and optimizes plant growth at a given location ${ }^{3-6}$. Opening of stomata, which is efficiently induced by blue light (BL; $390-500 \mathrm{~nm}$ ), is achieved by the swelling of guard cells that form the stomatal pore ${ }^{6}$. These specialized cells, in which all biological events required for stomatal opening, including light perception, $\mathrm{H}^{+}$-ATPase activation, $\mathrm{K}^{+}$uptake and swelling of guard cells, occur in a single cell, offer an excellent model system to investigate how light drives reversible cell expansion through changes in solute transport across the membranes.

BL is perceived by phototropins (phot1 and phot2), which comprise a small family of light-activated protein kinases associated with the plasma membrane ${ }^{7,8}$. Phototropins contain two photoreceptive domains called LOV1 (light, oxygen or voltage) and LOV2 close to the $\mathrm{N}$-terminus, and a C-terminal Ser/Thr kinase. Illumination of plant cells with BL results in phototropin autophosphorylation, an event that is prerequisite for phototropin-mediated responses such as phototropism, chloroplast movements, leaf flattening and stomatal opening ${ }^{8-10}$. However, the mechanisms by which phototropin transduces the light signal into downstream responses are largely unknown. In guard cells, the driving force for stomatal opening is generated by plasma membrane $\mathrm{H}^{+}$-ATPases that are activated via phototropins $^{11-13}$, and it has been established that protein phosphatase 1 (PP1) mediates the signalling between these two molecules ${ }^{14}$. However, the initial signalling event that couples photoreception to activation of the $\mathrm{H}^{+}$-ATPase has yet to be clarified. Genetic dissection of the components involved in this pathway has proven challenging as no reliable method exists to screen for mutants in BL-specific responses in guard cells.

We therefore developed a screening strategy to isolate Arabidopsis mutants defective in BL-dependent stomatal opening. Using this tool, we obtained mutants and demonstrate that a novel Ser/Thr protein kinase undergoes phosphorylation as an initial step of phototropin signalling.

\section{Results}

blus1 mutants lose stomatal responses to BL. Using an infrared thermograph ${ }^{15,16}$, we constructed a device to monitor changes in leaf temperature in response to $\mathrm{BL}$, and observed the expected temperature decreases resulting from transpiration. Application of weak BL $\left(5 \mu \mathrm{mol} \mathrm{m}^{-2} \mathrm{~s}^{-1}\right)$, superimposed on a background of red light (RL; $80 \mu \mathrm{mol} \mathrm{m}^{-2} \mathrm{~s}^{-1}$ ), decreased leaf temperature by $0.6^{\circ} \mathrm{C}$ in wild-type (WT) plants but not in the phot 1 phot 2 double mutant (Fig. 1a). This temperature decrease paralleled an increase in stomatal conductance triggered by BL (Fig. 1b). Consistent with the different photosensitivities reported previously ${ }^{11}$, phot 1 and phot 2 single mutants exhibited temperature decreases with low and high sensitivity to BL, respectively (Supplementary Fig. S1). This confirmed that the thermal imaging approach is valid for identifying mutants defective in BL-dependent stomatal opening.

We screened ethyl methanesulfonate-mutagenized lines and identified two allelic mutants. We named them as blus1-1 and -12 (blue light signaling1), because they shared characteristic phenotypes with a BL-induced slight increase of leaf temperature (Fig. 1c). Stomatal conductance in intact leaves gradually increased under strong RL $\left(600 \mu \mathrm{mol} \mathrm{m}^{-2} \mathrm{~s}^{-1}\right)$ and reached a steady state. Upon BL illumination $\left(5 \mu \mathrm{mol} \mathrm{m}{ }^{-2} \mathrm{~s}^{-1}\right)$, stomatal conductance increased rapidly in the WT and decreased when the illumination had ended (Fig. 1d); the blus1 mutants failed to exhibit this blue-light response. Stomata in the epidermis of blus1 mutants were unresponsive to $\mathrm{BL}$, but opened after the application of fusicoccin $(\mathrm{Fc})$, an activator of the $\mathrm{H}^{+}$-ATPase (Fig. 1e). Guard cell protoplasts from WT plants showed $\mathrm{H}^{+}$. pumping activity, demonstrated by the acidification of the medium in response to $\mathrm{BL}^{17}$. In contrast, blus1 mutant protoplasts slightly alkalized the medium under BL. Yet, similar acidification rates were induced in WT and blus 1 plants by $\mathrm{Fc}$ (Fig. 1f). Intriguingly, the plasma membrane $\mathrm{H}^{+}$-ATPase was phosphorylated in WT but not blus1 plants in response to BL (Fig. 1g), whereas similar $\mathrm{H}^{+}$-ATPase phosphorylation was found in WT and blus1 following Fc treatment (Fig. 1h). The activities of phot1 and phot2, as determined by a phosphorylation-dependent mobility shift assay, were not impaired in guard cells of blus1 (Fig. 1i). On the basis of these results, we concluded that blus 1 mutants were specifically defective in the signalling pathway between phototropins and plasma membrane $\mathrm{H}^{+}$-ATPases. It is also noteworthy that blus 1 mutants differ phenotypically from phot 1 phot 2 double mutant. blus1 mutants responded to $\mathrm{BL}$ by slight stomatal closure (Fig. 1c,d) and medium alkalization by guard cell protoplasts (Fig. 1f). These responses were absent in phot1 phot2 (refs 18,19), suggesting that a separate phototropin pathway that is independent of BLUS1 is present in guard cells, and may delay stomatal opening through some feedback mechanism.

BLUS1 encodes a novel protein kinase. Except for stomatal opening, blus1 mutants were not impaired in phototropinmediated responses, including phototropism, chloroplast movements and leaf flattening (Supplementary Fig. S2). Mutations in the BLUS1 gene were identified by map-based cloning and mapped to the central part of chromosome 4 (Fig. 2a). Sequencing of this region in blus1-1 and -1-2 mutants revealed that At4g14480, which encodes a novel Ser/Thr protein kinase, had point mutations at nucleotides 580 and 574, resulting in substitutions of Lys for Glu-194 and Thr for Ala-192, respectively (Fig. 2b). BLUS1 comprises 487 amino acids with a deduced molecular mass of $54.4 \mathrm{kDa}$, harbouring the E194K for blus1-1 and A192T for blus1-2 mutations within the kinase activation loop in the $\mathrm{N}$-terminus. We generated antibodies against the BLUS1 protein using the C-terminal region of BLUS1. Immunological analysis indicated that the missense mutation lines of blus 1-1 and -1-2 produced BLUS1 proteins that had lost signalling function, and that the T-DNA insertion lines of blus1-3 and blus1-4 were null mutants (Fig. 2c). Accordingly, these two mutants were defective in leaf temperature decreases and stomatal opening in response to BL (Fig. 2d,e). A GFP-BLUS1 transgene complemented the blus1-1 mutant phenotype and restored BLdependent decreases in leaf temperature (Fig. 2f). The subcellular localization of GFP:BLUS1 was investigated using complemented lines, in which GFP-BLUS1 was localized to the cytosol (Fig. 2g). Furthermore, RT-PCR analyses revealed that BLUS1 was expressed in guard but not mesophyll cells (Fig. 2h), which corroborates previous microarray data ${ }^{20}$.

BLUS1 phosphorylation is essential for stomatal opening. BLUS1 showed an electrophoretic mobility shift induced by BL illumination; this response was absent in phot-deficient mutant (Fig. 3a), suggesting that BLUS1 might be phosphorylated by the phototropins. To address the phosphorylation status of BLUS1, we collected phosphopeptides from guard cell protoplasts by hydroxy acid-modified metal oxide chromatography ${ }^{21,22}$ and analysed them by nanoLC-MS/MS. The amount of BLUS1 phosphopeptides increased after BL illumination (Fig. 3b). 
a
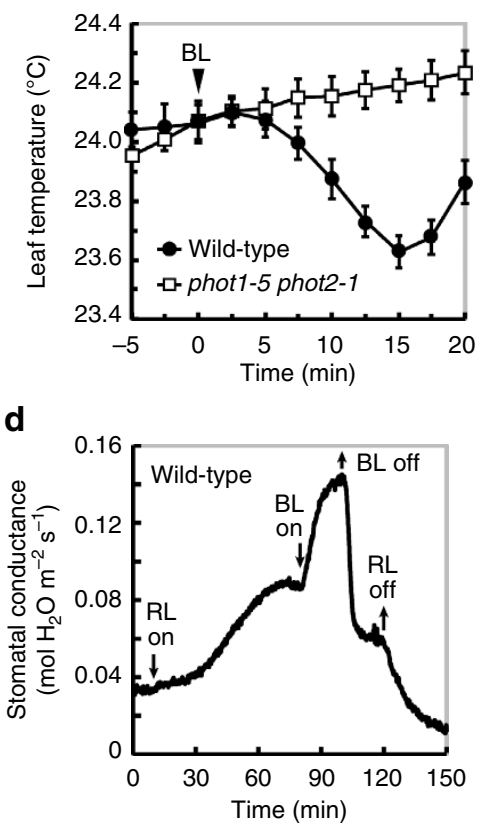

b

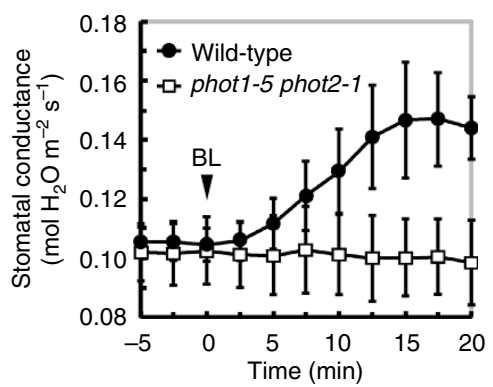

C

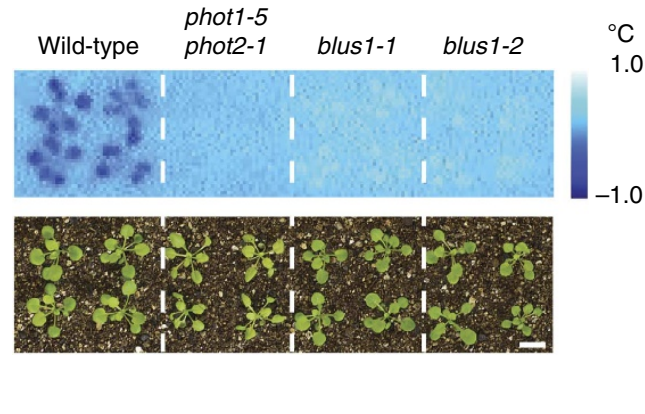

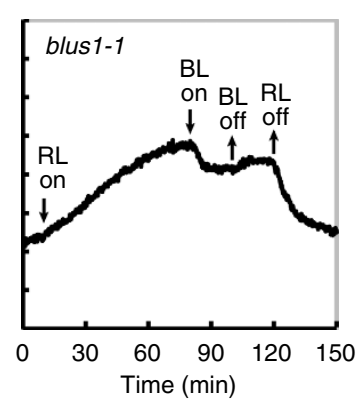

e

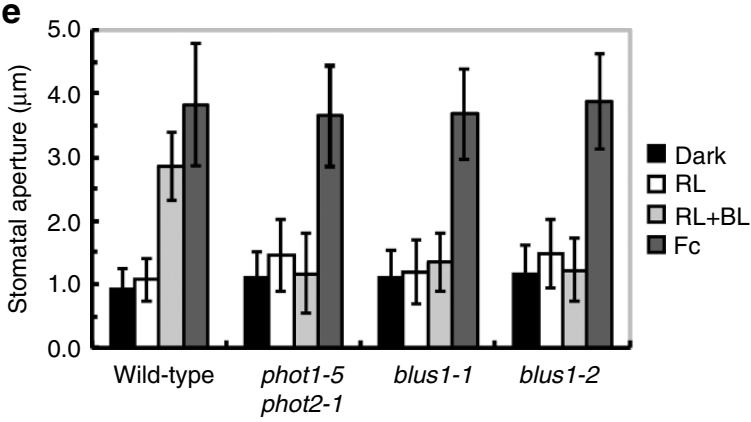

g
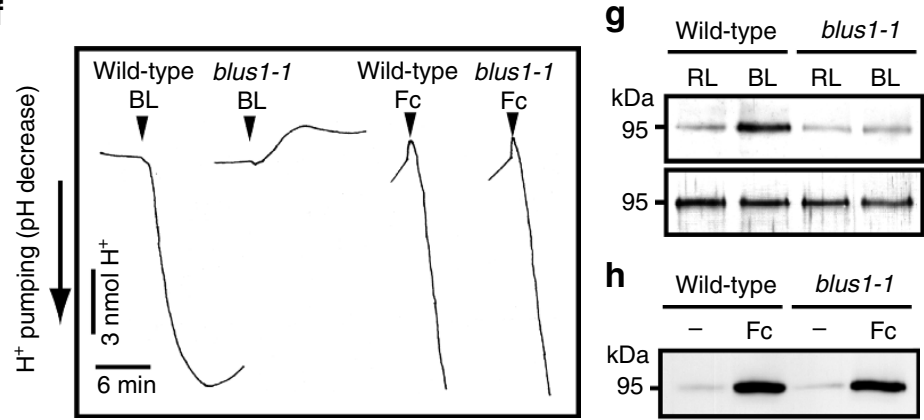

i

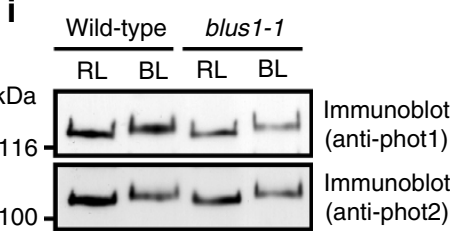

h

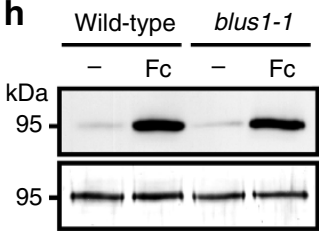

Protein blot (GST-14-3-3)

Immunoblot (anti- $\mathrm{H}^{+}$-ATPase)

100

Protein blot

(GST-14-3-3)

Immunoblot

(anti-H+-ATPase)

Figure 1 | Mutations in BLUS1 impair stomatal responses to BL. (a,b) Changes of leaf temperature (a) and stomatal conductance (b) in response to BL. Leaves were illuminated by RL $\left(80 \mu \mathrm{mol} \mathrm{m}{ }^{-2} \mathrm{~s}^{-1}\right)$ for $50 \mathrm{~min}$, and weak BL $\left(5 \mu \mathrm{mol} \mathrm{m}{ }^{-2} \mathrm{~s}^{-1}\right)$ was superimposed. Bars represent means $\pm \mathrm{s}$.e.m. $(n=5)$. (c) Thermal images of Arabidopsis leaves from WT plants, phot1 phot2 and blus1 mutants responding to BL. Images were obtained by subtracting an image taken before BL from one taken 15 min after BL. Lower panels show the plants. The bar represents $1 \mathrm{~cm}$. (d) Light-dependent changes in stomatal conductance of intact leaves from wild-type and blus1 mutant. RL $\left(600 \mu \mathrm{mol} \mathrm{m} \mathrm{m}^{-2} \mathrm{~s}^{-1}\right)$ and BL $\left(5 \mu \mathrm{mol} \mathrm{m}{ }^{-2} \mathrm{~s}^{-1}\right)$ were turned on and off as indicated. (e) Light- and Fc-dependent stomatal opening in the epidermis from WT and mutant plants. Epidermal strips from dark-adapted plants were illuminated with $\mathrm{RL}\left(50 \mu \mathrm{mol} \mathrm{m}{ }^{-2} \mathrm{~s}^{-1}\right)$ with or without BL $\left(10 \mu \mathrm{mol} \mathrm{m}{ }^{-2} \mathrm{~s}^{-1}\right)$ for $2 \mathrm{~h}$. Fc was added to the epidermis at $10 \mu \mathrm{M}$. Bars represent means $\pm \mathrm{s} . d$. $(n=75$, pooled from triplicate experiments). (f) BL- and Fc-dependent $\mathrm{H}^{+}$pumping in guard cell protoplasts from WT plants and blus1 mutants. Guard cell protoplasts were illuminated by RL $\left(600 \mu \mathrm{mol} \mathrm{m}{ }^{-2} \mathrm{~s}^{-1}\right)$ for $2 \mathrm{~h}$, and a pulse of BL $\left(100 \mu \mathrm{mol} \mathrm{m}{ }^{-2} \mathrm{~s}^{-1}, 30 \mathrm{~s}\right)$ was applied as indicated. Ten micromolar Fc was used. $(\mathbf{g}, \mathbf{h})$ BL- $(\mathbf{g})$ and Fc-dependent $(\mathbf{h})$ phosphorylation of the $\mathrm{H}^{+}$-ATPase in guard cell protoplasts. Phosphorylation of the $\mathrm{H}^{+}-\mathrm{ATPase}$ was determined by protein blotting using a 14-3-3 protein. Each lane contained 1.5 and $3.5 \mu$ g of guard cell proteins for protein blotting (upper panels) and immunoblotting (lower panels). (i) BL-dependent phosphorylation of phototropins (phot1, phot2). The autophosphorylation of phototropins was determined by the upward electrophoretic mobility shift using anti-phot 1 and anti-phot 2 antibodies. Each lane contained $5 \mu$ g of guard cell proteins.

Moreover, the analysis indicated that the light-induced phosphorylation of BLUS1 occurred at the Ser-348 residue in the C-terminus (Fig. 3c,d). Consequently, we investigated the physiological role of Ser-348 phosphorylation in BLUS1. The WT BLUS1 gene complemented the blus1 phenotype, but mutation of Ser-348 to Ala (S348A) failed to complement the mutant phenotype (Fig. 3e). Furthermore, a kinase-dead mutant of BLUS1 in which Asp-157 was replaced with Asn (D157N) ${ }^{23}$ did not rescue the blus1 phenotype (Fig. 3e). The production of the mutant proteins in the transgenic plants was independently verified (Supplementary Fig. S3). We concluded that phosphorylation of Ser-348 as well as BLUS1 kinase activity is essential for BLUS1 signalling. As Ser-348 and the kinase domain are located in the $\mathrm{C}$ - and the $\mathrm{N}$-terminus, respectively, the phosphorylation of Ser-348 may contribute to the regulation of kinase activity and/or to substrate recognition. Ser-348 is part of the consensus sequence motif, (RRISGWNF), which appears to be conserved in angiosperms, both monocots and dicots (Supplementary Fig. S4). BLUS 1 appears to be a single gene in many plant species. We note that no protein kinases with this motif could be found in a representative bryophyte (Physcomitella patens $)^{24}$ or lycophyte (Selaginella moellendorffii) ${ }^{25}$. 
a

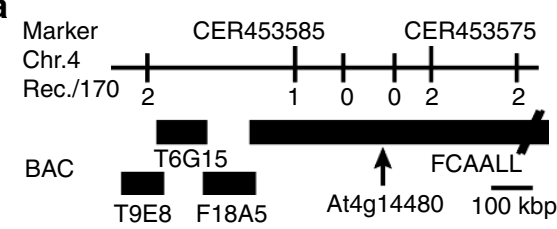

b

blus 1-3

(SALK_000221)

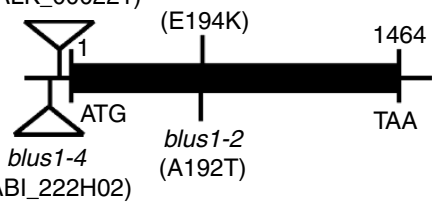

C

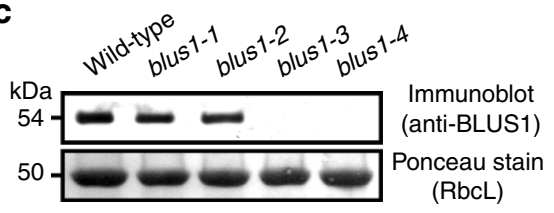

d

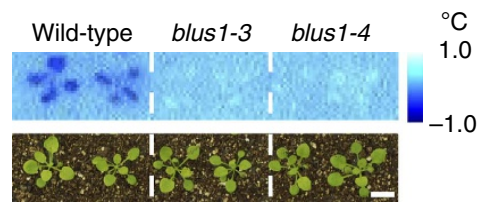

e

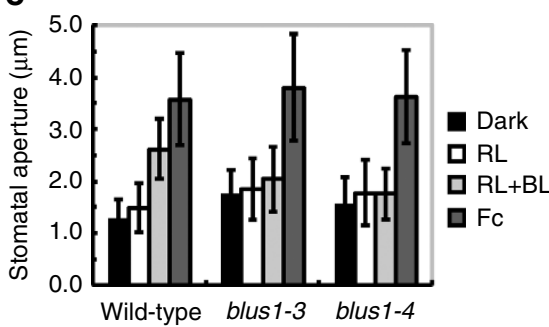

f

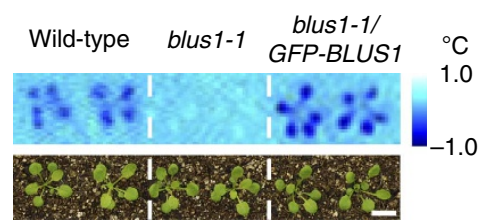

g

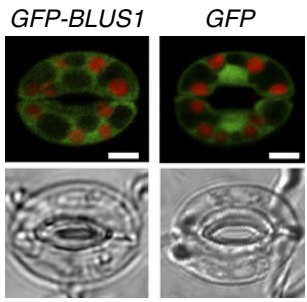

h

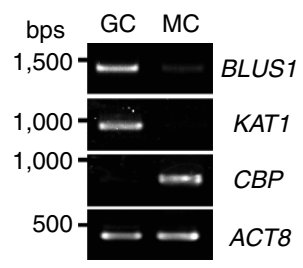

Figure 2 | Identification of the BLUS1 gene. (a) Map-based cloning of the BLUS1 gene. BLUS1 was mapped to the 290 kbp region between markers CER453585 and CER433575 on BAC clone FCAALL. Numbers indicate the recombination from a total of 170 chromosomes. (b) Genomic structure of the BLUS1 gene and sites of blus1 mutations. Black box indicates the protein-coding region. (c) Immunoblot analysis of BLUS1 protein in the blus1 mutants. The Rubisco large subunit (RbcL) was used as the loading control. Each lane contained $5 \mu \mathrm{g}$ of guard cell proteins. (d) Impairment of the BL-dependent leaf temperature decrease of the blus1 T-DNA insertion mutants. The bar represents $1 \mathrm{~cm}$. (e) Impairment of the BL-dependent stomatal opening in the epidermis of the T-DNA insertion mutants. Bars represent means \pm s.d. ( $n=75$, pooled from triplicate experiments). (f) Rescue of the leaf temperature decrease by the transformation of the blus1-1 mutant with GFP-BLUS1. (g) Confocal images of GFP (green) and chlorophyll (red) fluorescence in guard cells of transgenic plants expressing GFP-BLUS1 or GFP alone (upper panels). Lower panels show differential interference contrast images. The bars represent $5 \mu \mathrm{m}$. (h) Specific expression of BLUS1 in guard cell protoplasts (GC), as determined by RT-PCR analysis. MC, mesophyll cell protoplasts. KAT1 and CBP were used as guard cell and mesophyll cell markers, respectively. ACT8 served as a control.

a

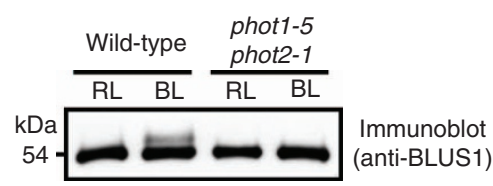

C

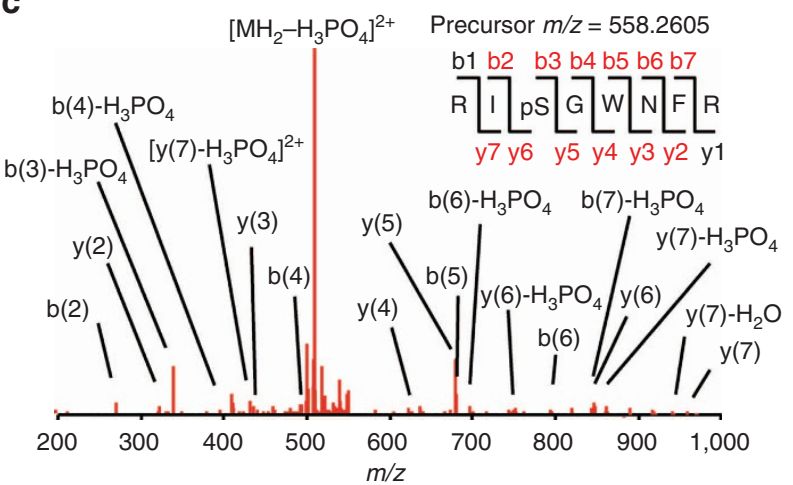

b

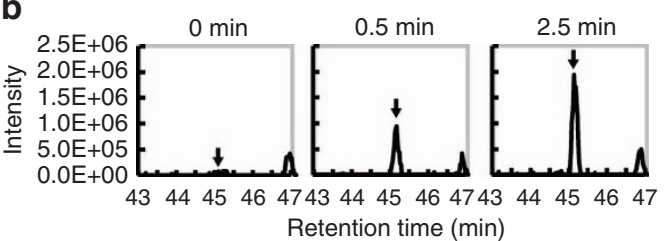

d

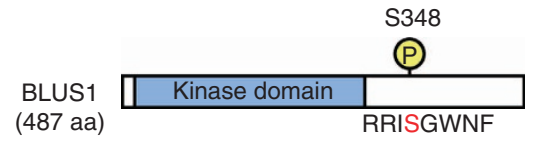

e

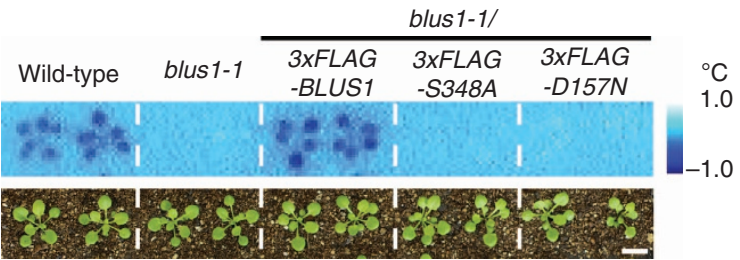

Figure 3 | The BL-dependent phosphorylation site in BLUS1. (a) BL-dependent electrophoretic mobility shift of BLUS1. The immunoblot was probed with anti-BLUS1 antibodies. Each lane contained $5 \mu$ g of guard cell proteins. (b,c) Phosphoproteome analyses of BL-dependent protein phosphorylation in guard cell protoplasts. Extracted ion chromatogram (b) and MS/MS spectrum (c) of the phosphopeptide corresponding to residues R346-R353 of BLUS1. (d) Schematic structure and phosphorylation site of BLUS1. RRISGWNF, a consensus motif around Ser-348. (e) Failure of BLUS1 (S348A) and BLUS1 $(D 157 N)$ to complement the defect in leaf temperature decrease in the blus1-1 mutant. The bar represents $1 \mathrm{~cm}$.

Phototropins phosphorylate BLUS1 as a primary event. We generated phosphospecific antibodies against pSer-348-BLUS1 (anti-pSer348) to examine BLUS1 phosphorylation more closely.
BL-dependent phosphorylation of Ser-348 was found in the WT but not in the phot 1 phot 2 double mutant (Fig. 4a). Ser-348 phosphorylation was also detected in phot 1 and phot 2 single 
a

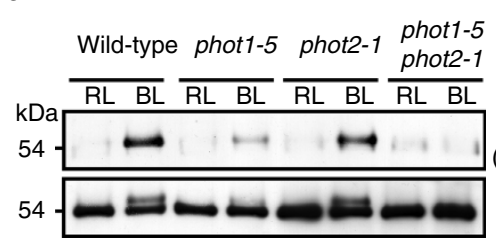

b

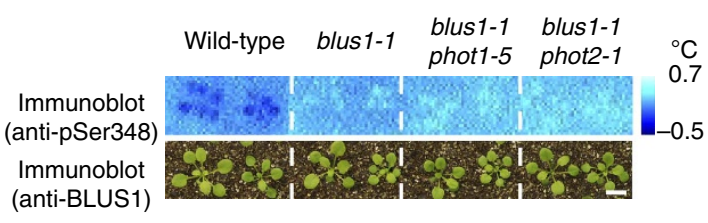

d

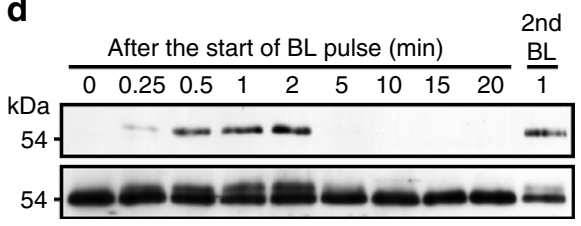

g e

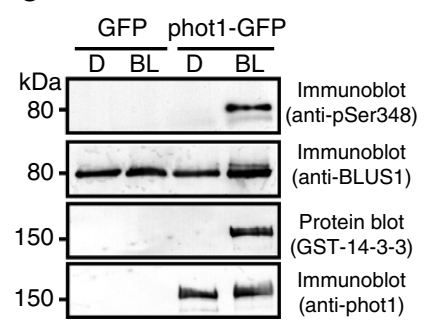

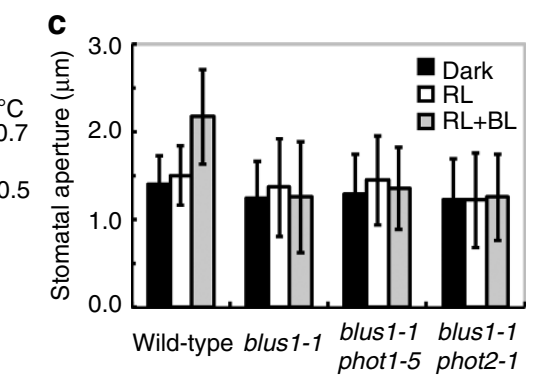

f

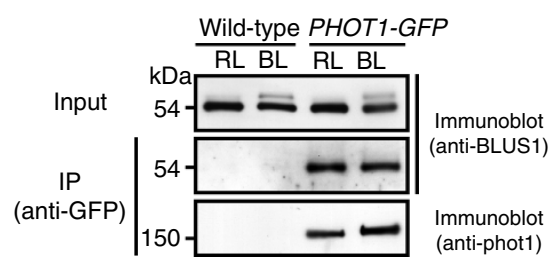

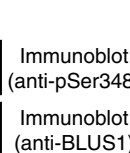
(anti-BLUS1)

(B1) $\frac{\text { P1N (1-293) }}{\text { P1C (620-996) }}$
phot1
(P1)

(P1) $\mathrm{P} 1 \mathrm{~N}(1-619)$

h

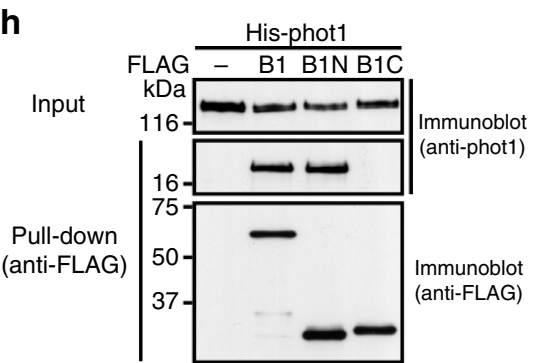

i

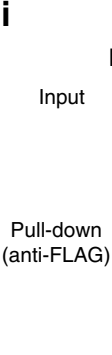

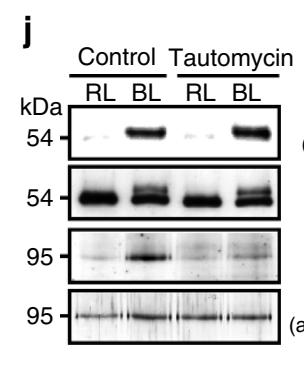

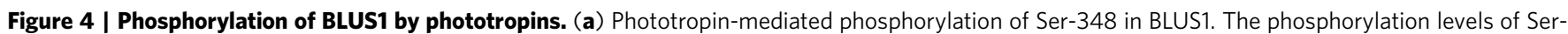
348 were determined by immunoblotting using anti-pS348 antibodies. The amounts of BLUS1 proteins were determined using anti-BLUS1 antibodies. Each lane contained $5 \mu \mathrm{g}$ of guard cell proteins. (b) Impairment of BL-dependent leaf temperature decrease in the double mutants blus1 phot1 and blus1 phot2. The bar represents $1 \mathrm{~cm}$. (c) Impairment of BL-dependent stomatal opening in the double mutants. Bars represent means \pm s.d. ( $n=75$, pooled from triplicate experiments). (d) Time course of BLUS1 phosphorylation levels on Ser-348 in response to BL. (e) Phosphorylation of BLUS1 on Ser-348 by phot1 in vitro. GST-BLUS1 was expressed in E. coli. phot1-GFP was immunopurified from etiolated seedlings that had been illuminated with BL or kept in darkness (D). GST-BLUS1 was incubated with phot1-GFP in the presence of ATP, and BLUS1 phosphorylation was measured using anti-pS348 antibodies.

Autophosphorylation of phot1 was detected by protein blotting using a 14-3-3 protein. Transgenic plants expressing GFP were used as a control. (f) Coimmunoprecipitation of BLUS1 with phot1 in guard cells. Guard cell protoplasts (100 $\mu$ g of proteins) expressing PHOT1-GFP were illuminated with RL, and then a pulse of BL was applied. The proteins were immunoprecipitated with an anti-GFP antibody, and the immunoprecipitate was probed with anti-BLUS1 or anti-phot1 antibodies. Wild-type plants were used as controls. (g) Schematic structures of BLUS1 and phot1 used in pull-down assays. B1N; N-terminal fragment of BLUS1 (amino acids 1-294), B1C; C-terminal fragment of BLUS1 (294-487), P1N; N-terminal fragment of phot1 (1-619), P1C; C-terminal fragment of phot1 (620-996). (h,i) Pull-down assays of phot1 and BLUS1. All proteins were synthesized by in vitro transcription/translation reactions. Indicated regions of FLAG-tagged BLUS1 were incubated with His-phot1 (h) and those of FLAG-tagged phot1 were incubated with His-BLUS1 (i). The proteins bound to anti-FLAG beads were detected using anti-phot1 (h), anti-BLUS1 (i) and anti-FLAG (h,i) antibodies. (j) Effect of $5 \mu M$ tautomycin on BL-dependent phosphorylation of BLUS1 and $\mathrm{H}^{+}$-ATPase. (k) Proposed pathway of BL signalling in guard cells.

mutants (Fig. 4a), though the phot1 mutant displayed a lower degree of phosphorylation. Furthermore, blus1 phot 1 and blus 1 phot 2 double mutants exhibited the same leaf temperature changes as the blus 1 single mutant (Fig. 4b). Stomatal opening in these double mutants showed the impaired responses typical of the blus 1 single mutant (Fig. 4c). These results indicated that signals from both phot 1 and phot 2 are transmitted to BLUS1.

We monitored the phosphorylation status of BLUS1 following a pulse of BL for $30 \mathrm{~s}$ at greater resolution. The phosphorylation of Ser-348 was initiated $15 \mathrm{~s}$ after the start of the pulse, reached a maximum within $1 \mathrm{~min}$, and had returned to the initial dephosphorylated state by $5 \mathrm{~min}$ (Fig. $4 \mathrm{~d}$ ), with a re-phosphorylation of
BLUS1 following a second pulse. The time course of BLUS1 phosphorylation revealed a more rapid response than described for $\mathrm{H}^{+}$-ATPase phosphorylation ${ }^{12,26}$ and coincided with the timing of phot1 autophosphorylation ${ }^{9,26}$. Thus, it appeared conceivable that phototropins directly phosphorylate BLUS1. To test this possibility, we assayed BLUS1 phosphorylation in vitro by incubating recombinant BLUS1 with functional phot1-GFP with high purity from etiolated Arabidopsis seedlings that had been illuminated with BL (Supplementary Fig. S5). In the presence of phot1-GFP, Ser-348 of BLUS1 became phosphorylated in a BL-dependent manner (Fig. 4e). Activation of phot1-GFP by BL was confirmed by 14-3-3 protein binding. 
Moreover, the BLUS1 protein co-immunoprecipitated with phot1-GFP from guard cells in the presence and absence of BL (Fig. 4f). Finally, we showed that photl directly interacted with BLUS1 in vitro by pull-down assays in which phot1 or BLUS1 proteins were used as bait (Fig. $4 \mathrm{~h}, \mathrm{i}$ ). These findings confirmed that BLUS1 is an endogenous substrate of phototropin kinase.

Next we investigated the site of interaction between phot 1 and BLUS1 using $\mathrm{N}$ - and C-terminal fragments of these two recombinant proteins (Fig. $4 \mathrm{~g}$ ). Full-length phot1 interacted with the BLUS1 N-terminus, which includes the kinase domain (Fig. 4h). Full-length BLUS1 interacted with both the $\mathrm{N}$ - and C-termini of phot1, including the photosensory LOV and kinase domains, respectively (Fig. 4i). Interestingly, immunoblot analysis indicated that the phot 1 kinase lacking the LOV domains (P1C) induced a mobility shift of BLUS1 (Fig. 4i) due to the phosphorylation of Ser-348 in the dark (Supplementary Fig. S6). In contrast, the full-length phot1 affected BLUS1 mobility only slightly (Fig. 4i). We concluded that the C-terminus containing the phot1 kinase phosphorylates BLUS1 at the physiological site, and that the LOV domains act as inhibitors of the kinase activity, as has been reported from a model system in which the LOV2 domain inhibits in vitro casein phophorylation by phot 2 kinase 27 .

As BLUS1 is a Ser/Thr protein kinase, it could be hypothesized to directly phosphorylate the $\mathrm{H}^{+}$-ATPase in guard cells. However, Fc induced $\mathrm{H}^{+}$-ATPase phosphorylation in blus1 mutants as well as WT plants (Fig. 1h), suggesting an involvement of protein kinases other than BLUS1. To identify the role of BLUS1 in this pathway, we tested the effects of tautomycin, an inhibitor of PP1, on the BL-dependent phosphorylation of BLUS1 and $\mathrm{H}^{+}$-ATPase. The inhibitor suppressed $\mathrm{H}^{+}$-ATPase phosphorylation, but had no effects on BLUS1 phosphorylation (Fig. 4j). BLUS1 therefore appears to precede PP1 in the signalling pathway for stomatal opening (Fig. 4k).
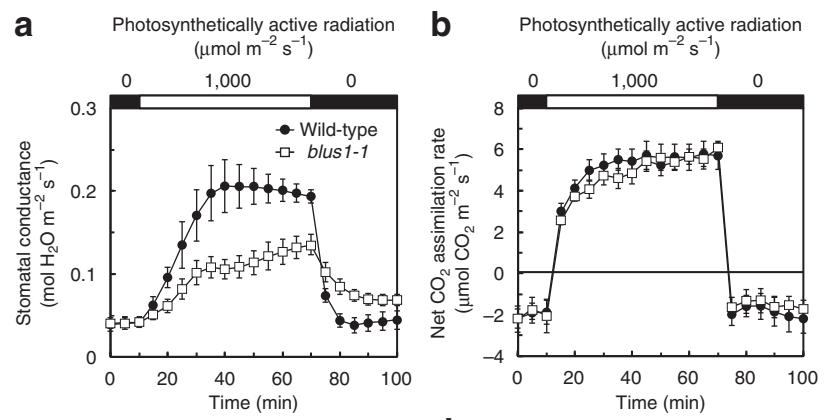

C Photosynthetically active radiation $\left(\mu \mathrm{mol} \mathrm{m} \mathrm{m}^{-2} \mathrm{~s}^{-1}\right)$
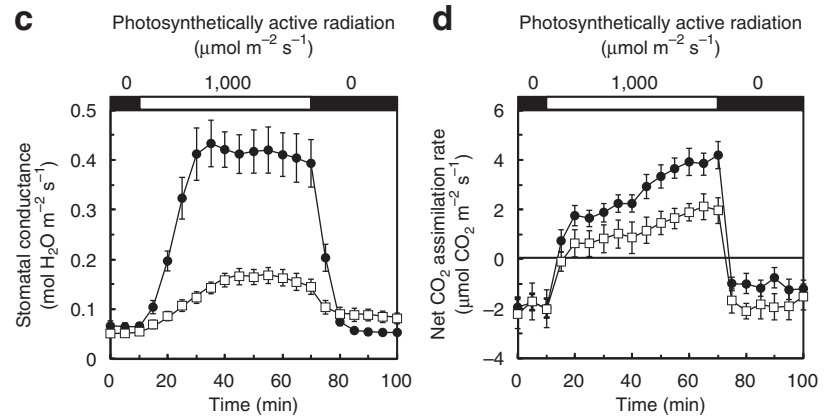

Figure 5 | blus1 mutants decrease stomatal conductance and $\mathrm{CO}_{2}$ fixation (a,c) Stomatal conductance in response to white light $\left(1,000 \mu \mathrm{mol} \mathrm{m}{ }^{-2} \mathrm{~s}^{-1}\right)$. The bars represent means \pm s.e.m. $(n=5) .(\mathbf{b}, \mathbf{d})$ Photosynthetic $\mathrm{CO}_{2}$ assimilation rate in response to white light. $(\mathbf{a}, \mathbf{b})$ 350 p.p.m. $\mathrm{CO}_{2}$. (c,d) 200 p.p.m. $\mathrm{CO}_{2}$. The bars represent means \pm s.e.m. $(n=5)$. blus1 mutants decrease stomatal conductance and $\mathrm{CO}_{2}$ fixation. As blus1 mutants are defective solely in BL-dependent stomatal opening (Fig. 1 and Supplementary Fig. S2), they offer an excellent system to assess how the stomatal BL response contributes to photosynthetic $\mathrm{CO}_{2}$ assimilation under natural light conditions. We compared the stomatal conductance and photosynthetic $\mathrm{CO}_{2}$ assimilation between WT and blus 1 under white light at $1,000 \mu \mathrm{mol} \mathrm{m}^{-2} \mathrm{~s}^{-1}$ and well-watered conditions. As expected, stomatal conductance was strongly reduced in blus 1 compared with the WT at 350 p.p.m. $\mathrm{CO}_{2}$ (Fig. 5a), but their rates of photosynthetic $\mathrm{CO}_{2}$ assimilation were indistinguishable (Fig. 5b). Upon transfer to darkness, blus1 mutants showed a slower decrease in conductance than WT plants and did not close stomata completely within $30 \mathrm{~min}$ (Fig. 5a). The conductance was enhanced significantly in the WT but only slightly increased in blus1 when the $\mathrm{CO}_{2}$ concentration was reduced to 200 p.p.m. (Fig. 5c); the net assimilation rate was two-fold higher in WT than in blus1 (Fig. 5d). The greater enhancement of stomatal aperture in response to decreased $\mathrm{CO}_{2}$ in the WT compared with blus 1 is partly due to that the $\mathrm{BL}$-specific response of stomata is stimulated by low intercellular concentration of $\mathrm{CO}_{2}$ (ref. 28).

\section{Discussion}

We demonstrated that the phosphorylation of Ser-348 in BLUS1 was essential for the activation of the $\mathrm{H}^{+}$-ATPase to drive stomatal opening, and concluded that BLUS1 was a substrate for phototropin kinase and that its phosphorylation was a primary event in phototropin signalling. Two potential substrates for phototropin kinase that are involved in phototropism have been identified, namely the auxin efflux transporter ATP-binding cassette B19 (ref. 29) and phytochrome kinase substrate 4 (ref. 30). However, neither the site of phosphorylation nor the nature of the role in phototropism is known for these two proteins. To our knowledge, BLUS1 is the first identified phototropin substrate with a known essential role in phototropin-mediated signalling.

Phototropins mediate a wide range of plant responses, including phototropism, chloroplast relocation, leaf flattening, leaf positioning and stomatal opening. It still remains an open question as to how phototropins bring about a diverse set of $\mathrm{BL}$ responses in plants $7,8,10$. We demonstrated that mutation of the BLUS1 gene does not affect any of the other phototropinmediated responses tested (Supplementary Fig. S2). Furthermore, no common components except phototropins have been found in the signalling pathways of phototropism, chloroplast movements and stomatal opening ${ }^{8}$. Apparently, several BL signalling pathways diverge from a component just downstream of the phototropins.

The blus1 mutant exhibits loss of stomatal BL response (Fig. 1) and shows slow and incomplete stomatal closure when the plants are transferred to darkness (Fig. 5a). These properties of blus 1 mutants resemble those of ferns that lack stomatal BL responses and exhibit slow and incomplete stomatal closure upon darkness ${ }^{2,31}$. As a large decrease in the atmospheric $\mathrm{CO}_{2}$ concentration occurred during the Devonian and Carboniferous periods, the time when seed plants evolved ${ }^{32}$, the acquisition of stomatal $\mathrm{BL}$ responses may have conferred competitive advantages over plants lacking these responses.

\section{Methods}

Plant materials and growth conditions. The Arabidopsis thaliana ecotype Columbia was used in all experiments. Ethyl methanesulfonate-mutagenized M2 seeds were purchased from Lehle Seeds or prepared by the Nisshoku Group. The phot1-5, phot 2-1 and phot1-5 phot2-1 mutants and transgenic PHOT1-GFP plants have been described elsewhere ${ }^{33-35}$. blus1-3 (SALK_000221) and blus1-4

(GABI_222H02) were obtained from the Nottingham Arabidopsis Stock Center (NASC), Nottingham, UK and GABI-Kat, respectively. 
For the mutant screen, plants were grown for 11 days on $0.8 \%(\mathrm{w} / \mathrm{v})$ agar plates containing half-strength Murashige-Skoog salts ( $\mathrm{pH}$ 5.7), $2.3 \mathrm{mM}$ MES and 1\% (w/ v) sucrose under continuous white light $\left(60 \mu \mathrm{mol} \mathrm{m}^{-2} \mathrm{~s}^{-1}\right)$ at $23^{\circ} \mathrm{C}$. The plants were then transferred to a soil:vermiculite (1:1) mixture and further grown under a $13 / 11 \mathrm{~h}$ light/dark cycle for 9 days. For some experiments, the plants were grown on soil:vermiculite (1:1) for 4 weeks under a 14/10 h light/dark cycle.

\section{Mutant screen by thermal imaging. Leaf temperature was determined by} infrared thermography (TVS-8500, NEC Avio Infrared Technologies). Plants were kept in the dark overnight at $21-24^{\circ} \mathrm{C}$ with $40-50 \%$ relative humidity and were then illuminated with RL $\left(80 \mu \mathrm{mol} \mathrm{m}^{-2} \mathrm{~s}^{-1}\right)$ for $50 \mathrm{~min}$. Then, a weak continuous BL $\left(5 \mu \mathrm{mol} \mathrm{m}^{-2} \mathrm{~s}^{-1}\right)$ was superimposed on the RL. The RL was obtained by passing light from a red fluorescent lamp (FL40S R, Panasonic) through a colour filter (no. 22, Tokyo Butai Showmei), and the BL was provided by a light-emitting diode (5050ML60-W/B). Temperature decreases were visualized using thermal images, which were obtained by the subtraction of an image taken before the start of BL irradiation from one taken after 15 min of BL irradiation using PE Professional software (NEC Avio Infrared Technologies).

Stomatal aperture and gas exchange. The stomatal aperture in the abaxial epidermis was measured by microscopic examination (Eclipse TS100; Nikon) ${ }^{11}$. Stomatal conductance and photosynthetic $\mathrm{CO}_{2}$ assimilation were determined using a gas-exchange system (LI-6400, Li-Cor) ${ }^{18}$.

Isolation of guard cell protoplasts. Guard cell protoplasts were prepared enzymatically from leaves ${ }^{19,36}$. A typical yield of guard cell protoplasts was $4.3 \times 10^{7}$ cells $\left(1,000 \mu\right.$ g proteins) per 5,000 leaves ${ }^{19}$. Measurement of $\mathbf{H}^{+}$pumping. BL- and Fc-dependent $\mathrm{H}^{+}$pumping were
determined with a glass $\mathrm{pH}$ electrode as described previously ${ }^{19}$. The reaction mixture $(0.8 \mathrm{ml})$ contained $0.125 \mathrm{mM}$ MES- $\mathrm{NaOH}(\mathrm{pH} 6.0), 1 \mathrm{mM} \mathrm{CaCl}_{2}, 0.4 \mathrm{M}$ mannitol, $10 \mathrm{mM} \mathrm{KCl}$ and guard cell protoplasts ( $50-80 \mu \mathrm{g}$ proteins).

Immunoblot and protein blot analyses. Guard cell protoplasts were illuminated with RL $\left(600 \mu \mathrm{mol} \mathrm{m}^{-2} \mathrm{~s}^{-1}\right)$ for $30 \mathrm{~min}$, and then a pulse of BL $\left(100 \mu \mathrm{mol} \mathrm{m} \mathrm{m}^{-2}\right.$ $\mathrm{s}^{-1}, 30 \mathrm{~s}$ ) was superimposed. To detect phototropins and BLUS1, the phosphorylation reaction was terminated at $2 \mathrm{~min}$ after the start of the BL pulse by adding trichloroacetic acid (TCA) to the guard cell protoplast suspension. For the $\mathrm{H}^{+}$ATPase, the phosphorylation was terminated at $3.5 \mathrm{~min}$ after the pulse as described previously ${ }^{12}$. Immunoblotting was performed according to Kinoshita and Shimazaki ${ }^{12}$, with slight modifications. We generated antibodies against the C-terminal region and phosphorylated Ser-348 of BLUS1 by immunizing rabbits with recombinant GST-BLUS1 (K294-G487) and a synthetic phospho-BLUS1 peptide (KNRRIpSGWNF), respectively. Antibodies against phot1, phot2 and $\mathrm{H}^{+}$-ATPase were described previously ${ }^{12,18,19}$. Phosphorylation levels of phot1 and the $\mathrm{H}^{+}$-ATPase were determined by protein blotting using GST-14-3-3 (refs $12,36)$.

Phosphoproteome analysis. TCA-treated guard cell proteins $(100 \mu \mathrm{g})$ were suspended in $0.1 \mathrm{M}$ Tris- $\mathrm{HCl}(\mathrm{pH} 8.0)$ containing $8 \mathrm{M}$ urea, protein phosphatase inhibitors and a protease inhibitor cocktail (Sigma-aldrich), and the suspension was sonicated for $5 \mathrm{~min}$. The proteins were reduced by dithiothreitol, alkylated with iodoacetamide and digested with Lys-C, followed by trypsin treatment ${ }^{37}$. The digested samples were desalted using stage tips with C18 Empore disk membranes $(3 \mathrm{M})^{38}$. The samples were enriched for phosphopeptides by hydroxy acid-modified metal oxide chromatography using lactic acid-modified titania ${ }^{21,22}$. Briefly, the digested samples were diluted with $0.1 \%$ TFA, $80 \%$ acetonitrile containing $300 \mathrm{mg} \mathrm{ml}^{-1}$ lactic acid (solution A), and then loaded to custom-made metal oxide chromatography tips preliminary equilibrated with solution A. After successive washing with solution $\mathrm{A}$, and then $0.1 \%$ TFA and $80 \%$ acetonitrile, the peptides were eluted with $0.5 \%$ piperidine. The phosphopeptide fractions were desalted and concentrated using a vacuum evaporator and then subjected to nanoLC-MS/MS analyses using LTQ-Orbitrap (Thermo Fisher Scientific) ${ }^{39}$. Peptides and proteins were identified by automated database searches using Mascot version 2.3 (Matrix Science) in the TAIR database (release 10), with a precursor mass tolerance of 3 p.p.m., a fragment ion mass tolerance of $0.8 \mathrm{Da}$, and strict trypsin specificity allowing for up to two missed cleavages. Cysteine carbamidomethylation was set as a fixed modification. Methionine oxidation serine, threonine and tyrosine phosphorylations were allowed as modifications.

Generation of transgenic plants. The $3,965 \mathrm{bp}$ genomic sequence, including the promoter, coding region and $3^{\prime}$-UTR region of BLUS1, was amplified using primers 5 -CGGTATCGATAAGCTTGCTTTAGGAATGTTGAAAGTATTC AGAG- $3^{\prime}$ and $5^{\prime}$-TAGAACTAGTGGATCCTCGCCTGTAGTCACTTGGCAC- ${ }^{\prime}$. The product was subcloned into the pBluescript II KS $(+)$ (Stratagene) using the In-Fusion system (Clontech). The GFP or $3 x F L A G$ sequence was inserted after the start codon of BLUS1, and the resulting GFP-BLUS1 or 3xFLAG-BLUS1 fragment was introduced into the HindIII/BamHI site of the pRI 101-AN (Takara). Each construct was transformed into blus1-1 plants using Agrobacterium tumefaciens.

Point mutations. Site-directed mutagenesis was performed using the QuikChange Site-Directed Mutagenesis Kit (Stratagene). The primers used were $5^{\prime}$-TAGTG AAGAATAGAAGAATCGCTGGGTGGAATTTCCGTGAAG- ${ }^{\prime}{ }^{\prime}$ and $5^{\prime}$-CTTCA CGGAAATTCCACCCAGCGATTCTTCTATTCTTCACTA- $3^{\prime}$ for BLUS1-S348A, and $5^{\prime}$-GATCCGTGAAGCTCGCTAATTTCGGAGTATCTGCA-3' ${ }^{\prime}$ and $5^{\prime}$-TGC AGATACTCCGAAATTAGCGAGCTTCACGGATC-3' for BLUS1-D157N.

Localization of GFP-BLUS1. Fluorescent images were obtained using a confocal laser-scanning microscope (Digital Eclipse C1; Nikon) ${ }^{14}$. The wavelengths of excitation and emission for GFP were $488 \mathrm{~nm}$ and $515-530 \mathrm{~nm}$, and those for chlorophyll fluorescence were 543 and $590 \mathrm{~nm}$ long-pass.

RT-PCR analysis. Total RNA was extracted from guard cell and mesophyll cell protoplasts using ISOGEN (Nippon Gene) and RNeasy (Qiagen). First-strand cDNA was synthesized from $1 \mu \mathrm{g}$ of total RNA using the SuperScript III First-strand Synthesis System (Invitrogen). The primer sets used for PCR were $5^{\prime}$-ATGGCTCGGAACAAGCTCGAG- $3^{\prime}$ and $5^{\prime}$-TTAACCCAAAACAC TATCTTTATCAGCAC- $3^{\prime}$ for BLUS1; $5^{\prime}$-ACTTTACGCCAGTGGTCGT ACAAC- $3^{\prime}$ and $5^{\prime}$-AAGGACTTCTGGGCACCTGAATCT- $3^{\prime}$ for ACT8; $5^{\prime}$-ATGT CGATCTCTTGGACTCG- $3^{\prime}$ and $5^{\prime}$-CTTGAAGCAAACTCTGAAGCAGCTC- $3^{\prime}$ for KAT1; $5^{\prime}$-ATGGGTCTTGAAGTTGGGTCCTTATG-3' $3^{\prime}$ and $5^{\prime}$-TGCAGA ACACTTCTAGGGTGTTTCTC-3' for CBP1.

In vitro phosphorylation assay. Full-length BLUS1 was subcloned into the pGEX2T (GE Healthcare) and introduced into E. coli strain Rosetta-gami B (Merck). The GST-BLUS1 was purified using glutathione-Sepharose (GE Healthcare). phot1-GFP was obtained by immunoprecipitation from of 3-day-old etiolated seedlings that had been illuminated with BL $\left(100 \mu \mathrm{mol} \mathrm{m}^{-2} \mathrm{~s}^{-1}\right)$ for $1 \mathrm{~min}$ or kept in darkness. 1,500 etiolated seedlings were ground in extraction buffer containing $50 \mathrm{mM}$ MOPS-KOH (pH 7.5), $2.5 \mathrm{mM}$ EDTA, $100 \mathrm{mM} \mathrm{NaCl}, 0.5 \mathrm{mM}$ PMSF, $10 \mu \mathrm{M}$ leupeptin, $2 \mathrm{mM}$ DTT, $10 \mathrm{mM} \mathrm{NaF}, 0.5 \mathrm{mM}$ ammonium molybdate and $100 \mathrm{nM}$ calyculin A. Following centrifugation at $10,000 \mathrm{~g}$ for $10 \mathrm{~min}$, the resulting supernatant was centrifuged at $100,000 \mathrm{~g}$ for $1 \mathrm{~h}$ and microsomal membranes were obtained as a pellet. The membranes ( $250 \mu \mathrm{g}$ proteins) were resuspended in extraction buffer with $0.1 \%$ Triton X-100, and kept on ice for $10 \mathrm{~min}$. After centrifugation at $10,000 \mathrm{~g}$ for $10 \mathrm{~min}$, the supernatant was used for immunoprecipitation using the GFP-Trap (Chromotek). After incubation for $1 \mathrm{~h}$, the beads were washed three times and immunopurified photl-GFP was used for phosphorylation assay. The phosphorylation of GST-BLUS1 by phot1-GFP on beads was performed in a reaction mixture containing $25 \mathrm{mM}$ Tris- $\mathrm{HCl}(\mathrm{pH}$ 7.4), $10 \mathrm{mM}$ $\mathrm{MgCl}_{2}$ and $100 \mu \mathrm{M} \mathrm{ATP}$ at $30^{\circ} \mathrm{C}$ for $2 \mathrm{~h}$ under BL $\left(100 \mu \mathrm{mol} \mathrm{m}^{-2} \mathrm{~s}^{-1}\right)$ or in darkness. The phosphorylated GST-BLUS1 proteins were subjected to immunoblotting using anti-pSer348 and anti-BLUS1 antibodies.

Co-immunoprecipitation assay. Guard cell protoplasts ( $100 \mu \mathrm{g}$ proteins) were mixed with an equal volume of medium containing $100 \mathrm{mM}$ MOPS-KOH ( $\mathrm{pH} 7.5$ ), $5 \mathrm{mM}$ EDTA, $200 \mathrm{mM} \mathrm{NaCl}, 1 \mathrm{mM}$ PMSF, $20 \mu \mathrm{M}$ leupeptin, $4 \mathrm{mM}$ DTT, $20 \mathrm{mM}$ $\mathrm{NaF}, 1 \mathrm{mM}$ ammonium molybdate, $200 \mathrm{nM}$ calyculin A and $0.4 \%$ Triton X-100, and kept on ice for $10 \mathrm{~min}$. Following centrifugation at $10,000 \mathrm{~g}$ for $10 \mathrm{~min}$, the supernatant was reacted with GFP-Trap for $1 \mathrm{~h}$. After washing the GFP-Trap three times, immunoprecipitated proteins were detected using anti-BLUS1 and anti-phot1 antibodies.

In vitro pull-down assay. His-tagged and FLAG-tagged proteins were synthesized using an in vitro transcription/translation system (BioSieg). The protein pair used for the assay was produced in a single tube. Fifty microliter of the synthesized proteins were mixed with $450 \mu \mathrm{l}$ binding buffer containing $20 \mathrm{mM}$ Tris- $\mathrm{HCl}(\mathrm{pH} 7.4), 140 \mathrm{mM}$ $\mathrm{NaCl}$ and $0.1 \%$ Triton X-100, and were kept on ice for $10 \mathrm{~min}$. After centrifugation at $10,000 \mathrm{~g}$ for $10 \mathrm{~min}$, the supernatants were mixed with anti-FLAG M2 agarose (Sigma-Aldrich), and incubated for $1 \mathrm{~h}$. After washing the beads three times, the bound proteins were subjected to immunoblotting using anti-photl and anti-BLUS1 antibodies. FLAG-tagged proteins were detected using anti-FLAG (Sigma-Aldrich) and TrueBlot secondary antibodies (Rockland Immunochemicals).

Phylogenetic analysis. All sequence data were obtained from the phytozome homepage (http://www.phytozome.net). Sequence alignment was performed using the ClustalW program with default parameters (gap opening penalty, 10; gap extension penalty, 0.2 ; delay divergent cutoff, $30 \%$ ). Phylogenetic analysis was done using the MEGA software, version 5, with the neighbour-joining method ${ }^{40}$. All positions containing gaps and missing data were eliminated from the data set. The number of bootstrap replicates was 1,000 . 


\section{References}

1. Hetherington, A. M. \& Woodward, F. I. The role of stomata in sensing and driving environmental change. Nature 424, 901-908 (2003).

2. McAdam, S. A. M. \& Brodribb, T. M. Stomatal innovation and the rise of seed plants. Ecol. Lett. 15, 1-8 (2012).

3. Schroeder, J. I., Allen, G. J., Hugouvieux, V., Kwak, J. M. \& Waner, D. Guard cell signal transduction. Annu. Rev. Plant Physiol. Plant Mol. Biol. 52, 627-658 (2001).

4. Fan, L. M., Zhao, Z. X. \& Assmann, S. M. Guard cells: a dynamic signaling model. Curr. Opin. Plant Biol. 7, 537-546 (2004).

5. Roelfsema, M. R. G. \& Hedrich, R. In the light of stomatal opening: new insights into 'the watergate'. N. Phytol. 167, 665-691 (2005).

6. Shimazaki, K., Doi, M., Assmann, S. M. \& Kinoshita, T. Light regulation of stomatal movement. Annu. Rev. Plant Biol. 58, 219-247 (2007).

7. Briggs, W. R. \& Christie, J. M. Phototropins 1 and 2: versatile plant blue-light receptors. Trends Plant Sci. 7, 204-210 (2002).

8. Christie, J. M. Phototropin blue-light receptors. Annu. Rev. Plant Biol. 58, 21-45 (2007).

9. Inoue, S. et al. Blue light-induced autophosphorylation of phototropin is a primary step for signaling. Proc. Natl Acad. Sci. USA 105, 5626-5631 (2008)

10. Inoue, S., Takemiya, A. \& Shimazaki, K. Phototropin signaling and stomatal opening as a model case. Curr. Opin. Plant Biol. 13, 587-593 (2010).

11. Kinoshita, T. et al. Phot 1 and phot 2 mediate blue light regulation of stomatal opening. Nature 414, 656-660 (2001).

12. Kinoshita, T. \& Shimazaki, K. Blue light activates the plasma membrane $\mathrm{H}^{+}$. ATPase by phosphorylation of the C-terminus in stomatal guard cells. $E M B O J$ 18, 5548-5558 (1999)

13. Merlot, S. et al. Constitutive activation of a plasma membrane $\mathrm{H}^{+}$-ATPase prevents abscisic acid-mediated stomatal closure. EMBO J 26, 3216-3226 (2007).

14. Takemiya, A., Kinoshita, T., Asanuma, M. \& Shimazaki, K. Protein phosphatase 1 positively regulates stomatal opening in response to blue light in Vicia faba. Proc. Natl Acad. Sci. USA 103, 13549-13554 (2006).

15. Omasa, K., Hashimoto, Y. \& Aiga, I. A quantitative analysis of the relationship between $\mathrm{SO}_{2}$ or $\mathrm{NO}_{2}$ sorption and their acute effects on plant leaves using image instrumentation. Environ. Control Biol. 19, 59-67 (1981).

16. Merlot, S. et al. Use of infrared thermal imaging to isolate Arabidopsis mutants defective in stomatal regulation. Plant J 30, 601-609 (2002).

17. Shimazaki, K., Iino, M. \& Zeiger, E. Blue light-dependent proton extrusion by guard-cell protoplasts of Vicia faba. Nature 319, 324-326 (1986).

18. Doi, M., Shigenaga, A., Emi, T., Kinoshita, T. \& Shimazaki, K. A transgene encoding a blue-light receptor, phot 1 , restores blue-light responses in the Arabidopsis phot 1 phot 2 double mutant. J. Exp. Botany 55, 517-523 (2004).

19. Ueno, K., Kinoshita, T., Inoue, S., Emi, T. \& Shimazaki, K. Biochemical characterization of plasma membrane $\mathrm{H}^{+}$-ATPase activation in guard cell protoplasts of Arabidopsis thaliana in response to blue light. Plant. Cell. Physiol. 46, 955-963 (2005).

20. Leonhardt, N. et al. Microarray expression analyses of Arabidopsis guard cells and isolation of a recessive abscisic acid hypersensitive protein phosphatase $2 \mathrm{C}$ mutant. Plant Cell 16, 596-615 (2004).

21. Sugiyama, N. et al. Phosphopeptide enrichment by aliphatic hydroxy acidmodified metal oxide chromatography for nano-LC-MS/MS in proteomics applications. Mol. Cell Proteomics 6, 1103-1109 (2007)

22. Kyono, Y., Sugiyama, N., Imami, K., Tomita, M. \& Ishihama, Y. Successive and selective release of phosphorylated peptides captured by hydroxy acid-modified metal oxide chromatography. J. Proteome Res. 7, 4585-4593 (2008).

23. Hanks, S. K. \& Hunter, T. The eukaryotic protein kinase superfamily: kinase (catalytic) domain structure and classification. FASEB J 9, 576-610 (1995).

24. Rensing, S. A. et al. The Physcomitrella genome reveals evolutionary insights into the conquest of land by plants. Science 319, 64-69 (2008)

25. Banks, J. A. et al. The Selaginella genome identifies genetic changes associated with the evolution of vascular plants. Science 332, 960-963 (2011)

26. Kinoshita, T. et al. Blue-light- and phosphorylation-dependent binding of a 14-3-3 protein to phototropins in stomatal guard cells of broad bean. Plant. Physiol. 133, 1453-1463 (2003).

27. Matsuoka, D. \& Tokutomi, S. Blue light-regulated molecular switch of Ser/Thr kinase in phototropin. Proc. Natl Acad. Sci. USA 102, 13337-13342 (2005)

28. Assmann, S. M. Enhancement of the stomatal response to blue light by red light, reduced intercellular concentrations of $\mathrm{CO}_{2}$, and low vapor pressure differences. Plant Physiol. 87, 226-231 (1988).
29. Christie, J. M. et al. Phot1 inhibition of ABCB19 primes lateral auxin fluxes in the shoot apex required for phototropism. PLoS Biol. 9, e1001076 (2011).

30. Demarsy, E. et al. Phytochrome kinase substrate 4 is phosphorylated by the phototropin 1 photoreceptor. EMBO J 31, 3457-3467 (2012).

31. Doi, M., Wada, M. \& Shimazaki, K. The fern Adiantum capillus-veneris lacks stomatal response to blue light. Plant Cell. Physiol. 47, 748-755 (2006).

32. Beerling, D. J. \& Woodward, F. I. Vegetation and the Terrestrial Carbon Cycle: Modeling the First 400 Million Years (Cambridge University Press, Cambridge, UK, 2001).

33. Huala, E. et al. Arabidopsis NPH1: a protein kinase with a putative redoxsensing domain. Science 278, 2120-2123 (1997).

34. Kagawa, T. et al. Arabidopsis NPL1: a phototropin homolog controlling the chloroplast high-light avoidance response. Science 291, 2138-2141 (2001).

35. Sakamoto, K. \& Briggs, W. R. Cellular and subcellular localization of phototropin 1. Plant Cell 14, 1723-1735 (2002).

36. Takemiya, A., Yamauchi, S., Yano, T., Ariyoshi, C. \& Shimazaki, K. Identification of a regulatory subunit of protein phosphatase 1 which mediates blue light signaling for stomatal opening. Plant Cell. Physiol. 54, 24-35 (2013)

37. Saito, H., Oda, Y., Sato, T., Kuromitsu, J. \& Ishihama, Y. Multiplexed twodimensional liquid chromatography for MALDI and nanoelectrospray ionization mass spectrometry in proteomics. J. Proteome Res. 5, 1803-1807 (2006).

38. Rappsilber, J., Ishihama, Y. \& Mann, M. Stop and go extraction tips for matrixassisted laser desorption/ionization, nanoelectrospray, and LC/MS sample pretreatment in proteomics. Anal. Chem. 75, 663-670 (2003).

39. Nakagami, H. et al. Large-scale comparative phosphoproteomics identifies conserved phosphorylation sites in plants. Plant Physiol. 153, 1161-1174 (2010).

40. Tamura, K. et al. MEGA5: Molecular evolutionary genetics analysis using maximum likelihood, evolutionary distance, and maximum parsimony methods. Mol. Biol. Evol. 28, 2731-2739 (2011).

\section{Acknowledgements}

We are grateful to Drs T. Matsushita (Kyushu University) and T. Takami (Okayama University) for their valuable suggestions concerning mutant screening, and F. Oba and M. Aibe for their helpful technical assistance. We are also grateful to the Salk Institute Genomic Analysis Laboratory for providing the sequence-indexed Arabidopsis T-DNA insertion mutants, and the NASC and GABI-KAT for providing the seeds used in this work. This work was supported by JSPS KAKENHI Grant number 21227001 (to K.S.), MEXT KAKENHI Grant number 23120521 and 25120719, the Research Foundation for Opto-Science and Technology, and the Sumitomo Foundation (to A.T.)

\section{Author contributions}

A.T. and K.S. conceived and designed the experiments. A.T. performed most of the experiments and N.S. did the mass spectrometry analysis. H.F., T.T., S.Y. and A.H. carried out the mutant screening or prepared guard cell protoplasts. Y.T. assisted in in vitro transcription/translation experiments. A.T., N.S., J.M.C. and K.S. Analysed data and wrote the manuscript.

\section{Additional information}

Supplementary Information accompanies this paper at http://www.nature.com/ naturecommunications

Competing financial interests: The authors declare no competing financial interests.

Reprints and permission information is available online at http://npg.nature.com/ reprintsandpermissions/

How to cite this article: Takemiya A. et al. Phosphorylation of BLUS1 kinase by phototropins is a primary step in stomatal opening. Nat. Commun. 4:2094 doi: $10.1038 /$ ncomms3094 (2013).

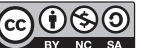

This work is licensed under a Creative Commons AttributionNonCommercial-ShareAlike 3.0 Unported License. To view a copy of this license, visit http://creativecommons.org/licenses/by-nc-sa/3.0/ 